\title{
39 FACTORS INFLUENCING ABNORMAL GLAND MORPHOGENESIS IN COLORECTAL CANCER (CRC)-TRANSLATIONAL STUDIES
}

R Topley, I Jagan, R K Deevi, A Fatehullah, J Eves, M Stevenson, M Loughrey, K Arthur, F C Campbell Centre for Cancer Research and Cell Biology, Queen's University of Belfast, Northern Ireland

\subsection{6/gutjnl-2013-305143.39}

Introduction Disruption of colorectal gland formation characterizes high grade, aggressive CRCs but causal mechanisms remain unclear. Glandular morphogenesis can be modelled in three-dimensional (3D) culture systems that enable investigation of specific oncogenic signals. We have shown that the tumour suppressor PTEN regulates 3D glandular morphogenesis in a Caco-2 colorectal organotypic model system through effects on the Rho-GTPase cdc42. Cdc42 is activated by specific guanine nucleotide exchange factors (GEFs) and influences gland lumen formation by regulation of apical membrane (AM) assembly. PTEN knockdown inhibits cdc42, disrupts AM integrity and induces a vacuolar, multilumen glandular phenotype evocative of high grade CRC. While PTEN has catalytically -active or -inactive functional domains relevant to phosphatidylinositol 3-kinase (PI3K) activity, Caco-2 gland development was unaffected by PI3K signalling.

Aims/Background Study aims were to investigate effects of PTEN PI3K catalytically -active or -inactive functional domains on 3D Caco-2 morphogenesis and to assess model fidelity to human CRC.

Method We used wild type PTEN-expressing Caco-2 cells and isogenic stable PTEN knockdown Caco-2 (KD) clones in two- (2D) and three-dimensional (3D) cultures as model 
systems. Cell membrane localization of specific cdc42 GEFs was investigated by cell fractionation and immunoblot. Effects of catalytically -active or -inactive PTEN mutants on cdc42 activity and/or AM integrity during 3D morphogenesis were investigated by transfection and confocal microscopy. Apical membrane integrity was assessed in human CRC by semiquantitative score of the AM marker, NHERF-1. CRC gland morphology was assessed by a validated grading system.

Results PTEN expression enhanced cell membrane recruitment of cdc42 GEFs with a specific role in 3D morphogenesis (Tuba, ITSN2). PTEN mutants containing an intact catalytically-inactive C2 domain enhanced cdc42 activity, restored AM integrity and rescued defective morphogenesis of 3D PTEN-KD Caco-2 cultures. Conversely, a C2 domain construct mutated at its CBR3 lipid-binding motif was ineffective. Fundamental attributes of the model system viz, associations between AM integrity and gland morphology were conserved and had prognostic significance in human CRC.

Conclusion PTEN deficiency impairs GEF membrane recruitment, cdc42 activation, apical membrane assembly and CRC glandular morphogenesis in a predictive colorectal cancer model system. Dissection of these networks may identify molecular targets for novel therapy, aimed at high grade CRC. 